\title{
Tunable spatial-frequency-shift 3D nanoscopy with chip compatibility overcomes the diffraction limit of the linear optical imaging system
}

\author{
Ning Dai ${ }^{*}$ \\ Shanghai Institute of Technical Physics, Chinese Academy of Sciences, Shanghai 200083, China
}

Received May 11, 2021; accepted May 18, 2021; published online June 2, 2021

Citation: N. Dai, Tunable spatial-frequency-shift 3D nanoscopy with chip compatibility overcomes the diffraction limit of the linear optical imaging system, Sci. China-Phys. Mech. Astron. 64, 294231 (2021), https://doi.org/10.1007/s11433-021-1719-y

The superresolution microscopy that broadens the detection range in the spatial frequency domain through the spatialfrequency-shift (SFS) effect [1-5] shows intriguing advantages including a large field of view, high speed, and good modularity. Significantly, it captures pictures in the wide-field mode and allows a universal implementation without using special fluorophores labeling. To enable the sub-diffraction resolution in the SFS microscopy, it is essential to use the evanescent wave illumination with a large wave vector, which can be built on the integrated photonic configuration, paving the way for a compact nanoscopy on a thin chip [6-8]. However, the lack of an approach to actively tune the SFS value in a large range hampers the progress to achieve deep-subwavelength resolution through evanescent illumination with ultra-large wave vectors. When the wave vector of the illumination surpasses twice the cutoff frequency of the objective, a missing band exists between the shifted components and the zeroth component in the spatial frequency domain, which causes severe artifacts and a distortion in the image. As a result, the resolution of current linear SFS microscopy has been restricted less than thrice the Abbe diffraction limit of the detection system.

Recently published in SCIENCE CHINA Physics, Mechanics \& Astronomy, the paper by Liu et al. [9] proposes a broadly tunable large SFS effect and a chip-compatible 3D

\footnotetext{
*Corresponding author (email: ndai@mail.sitp.ac.cn)
}

nanoscopy. With their method, the resolution of the linear optical system can be freely improved using an illumination chip of a larger effective refractive index without a theoretical limit. In the lateral dimension, the spatial-frequencyshift tunability is realized by modulating the azimuthal propagation direction of two evanescent waves, as shown in Figure 1(a) and (b). The effective wave vector of their superposition can be tuned actively and broadly to enable a wide-range and a complete detection in the spatial frequency domain, as shown in Figure 1(c). In the vertical dimension, a sectional saturation effect by intensity modulation is used to tune the vertical spatial frequency spectrum of the evanescent illumination to solve the vertical spatial distribution within the evanescent penetration depth. Finally, a 3D superresolution image can be achieved by multiplying the lateral and vertical distributions.

Liu et al. [9] selected $\mathrm{GaP}$ as the waveguide material; they considered its high refractive index and low optical loss in the visible spectrum. They designed the side length and the thickness of the decagonal $\mathrm{GaP}$ waveguide (Figure 1) to be 100 and $250 \mathrm{~nm}$, respectively. The effective refractive index of the TM fundamental mode was 3.38 at a wavelength of $561 \mathrm{~nm}$. A comparatively low NA of 0.9 was used to show the benefit of STUN for acquiring both a high resolution and a large FOV. Liu et al. [9] have demonstrated that the tunability of the SFS value is essential for the image reconstruction in a large-SFS nanoscopy. Otherwise, the 

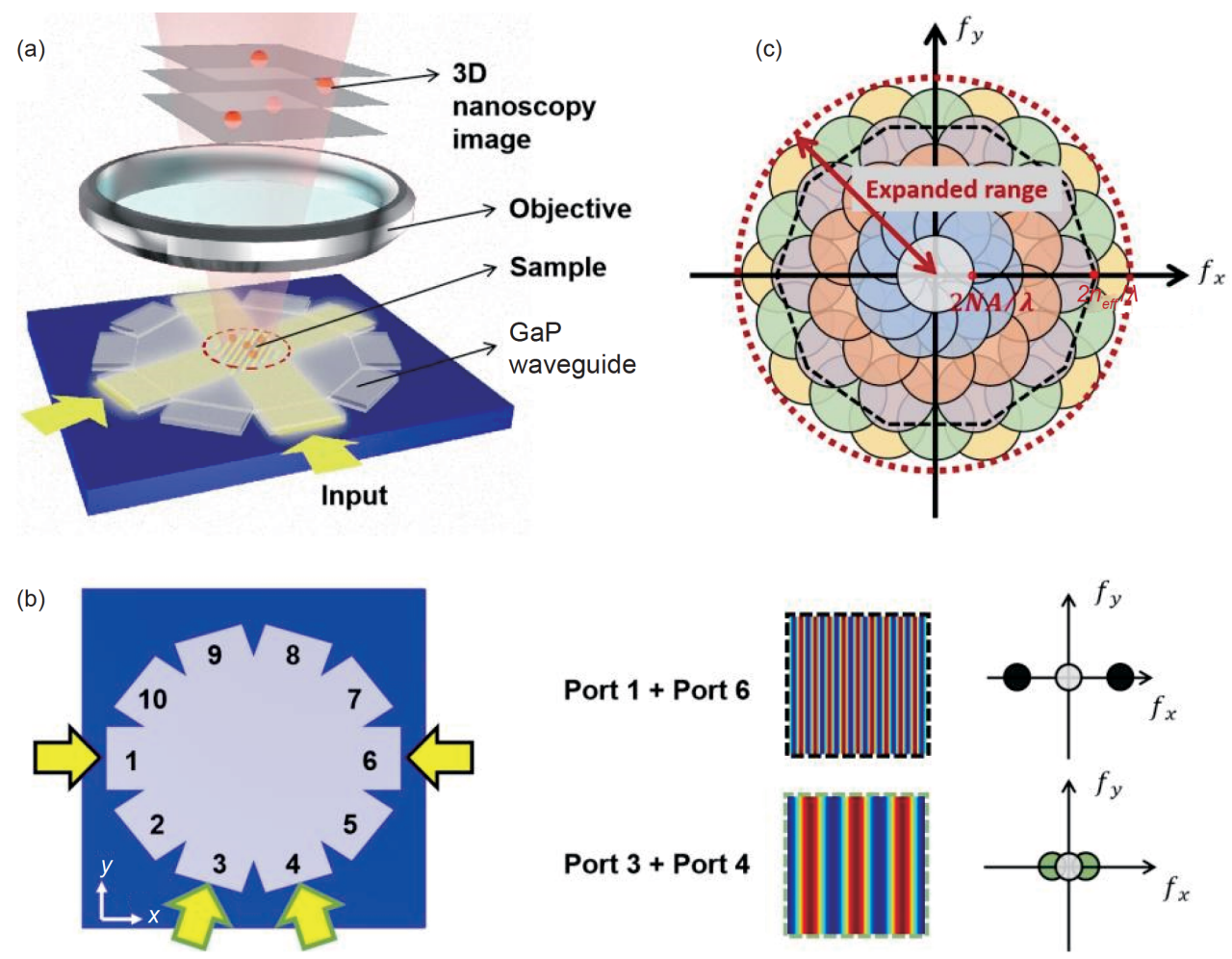

Figure 1 (Color online) Chip-compatible large and tunable SFS nanoscopy. (a) Schematic setup of a STUN chip based on GaP decagon waveguide; (b) two illumination patterns formed by the interference between the light modes from different waveguide input ports. The corresponding detection range in the spatial frequency domain is shown on the right. (c) The expanded detectable spatial frequency range in the tunable large SFS microscopy. $n_{\text {eff }}$ is the effective refractive index of the light mode in the waveguide.

missing band in the frequency domain will hinder the adoption of classical correlation-based algorithms from estimating the period, direction, and phases of the illumination patterns. Those parameters are necessary for solving and shifting back the high spatial frequency components. In their simulation, a nano-web 3D object was used to demonstrate the 3D imaging capability of STUN. One hundred and five raw images with excitation intensities that were significantly below the damage threshold of the biological sample in vivo were used to solve the $3 \mathrm{D}$ spatial distribution within the $30 \mathrm{~nm}$ penetration depth of the evanescent illumination.

Finally, a lateral resolution of $\lambda / 9$, which was five times the diffraction-limited resolution, and a vertical localization precision of $<\lambda / 200$ were achieved (detection objective $\mathrm{NA}=0.9$ ). Compared with the traditional illumination microscope without spatial-frequency-shift tunability, this method has a great improvement in resolution and better anti-noise capability.

In summary, this research reports a wide-range three-dimensional tunable deep SFS imaging method that is compatible with photonic chips, which makes the resolution enhancement of SFS imaging fundamentally eliminate the limitation of the detection aperture. This method is also a significant complement to the Nobel-prize-awarded superresolution techniques that rely on labeling the sample with fluorophores of nonlinear properties and require a point-by- point scanning process or the capturing of thousands of raw images. Compared with traditional superresolution microscopy imaging technology, this method is based on on-chip waveguide illumination and has the advantages of integration, low cost, and high stability. It can be further integrated with microfluidic and optoelectronic functional chips, providing a comprehensive research platform for the study of modern biological problems.

1 M. Tang, X. Liu, Z. Wen, F. Lin, C. Meng, X. Liu, Y. Ma, and Q. Yang, Laser Photon. Rev. 14, 1900011 (2020).

2 C. Pang, J. Li, M. Tang, J. Wang, I. Mela, F. Ströhl, L. Hecker, W. Shen, Q. Liu, X. Liu, Y. Wang, H. Zhang, M. Xu, X. Zhang, X. Liu, Q. Yang, and C. F. Kaminski, Adv. Funct. Mater. 29, 1900126 (2019).

3 X. Liu, C. Kuang, X. Hao, C. Pang, P. Xu, H. Li, Y. Liu, C. Yu, Y. Xu, D. Nan, W. Shen, Y. Fang, L. He, X. Liu, and Q. Yang, Phys. Rev. Lett. 118, 076101 (2017).

4 A. Bezryadina, J. Zhao, Y. Xia, X. Zhang, and Z. Liu, ACS Nano 12, 8248 (2018).

5 F. Wei, and Z. Liu, Nano Lett. 10, 2531 (2010).

6 Ø. I. Helle, F. T. Dullo, M. Lahrberg, J. C. Tinguely, O. G. Hellesø, and B. S. Ahluwalia, Nat. Photonics 14, 431 (2020), arXiv: 1903.05512.

7 X. Xu, X. Liu, C. Pang, Y. Ma, C. Meng, J. Zhang, X. Liu, and Q. Yang, J. Phys. D-Appl. Phys. 52, 284002 (2019).

8 M. Tang, Y. Han, D. Ye, Q. Zhang, C. Pang, X. Liu, W. Shen, Y. Ma, C. F. Kaminski, X. Liu, and Q. Yang, arXiv: 2103.09321.

9 X. Liu, M. Tang, C. Meng, C. Pang, C. Kuang, W. Chen, C. F. Kaminski, Q. Yang, and X. Liu, Sci. China-Phys. Mech. Astron. 64, 294211 (2021). 